\title{
Enquête
}

Archives de la revue Enquête

4 | 1996

La ville des sciences sociales

\section{The Kalela Dance / La danse du kalela}

Aspects des relations sociales chez les citadins africains en Rhodésie du Nord

The Kalela Dance. Aspects of Social Relationships among Urban Africans in Northern Rhodesia

\section{J. Clyde Mitchell}

Traducteur : Michel Agier et Stéphane Nahrath

\section{(2) OpenEdition}

Journals

Édition électronique

URL : http://journals.openedition.org/enquete/933

DOI : 10.4000/enquete.933

ISSN : 1953-809X

Éditeur :

Cercom, Éditions Parenthèses

Édition imprimée

Date de publication : 1 novembre 1996

Pagination : 213-243

Référence électronique

J. Clyde Mitchell, «The Kalela Dance / La danse du kalela », Enquête [En ligne], 4 | 1996, mis en ligne le

12 juillet 2013, consulté le 01 mai 2019. URL : http://journals.openedition.org/enquete/933; DOI :

10.4000/enquete.933

Ce document a été généré automatiquement le 1 mai 2019. 


\section{The Kalela Dance / La danse du kalela}

Aspects des relations sociales chez les citadins africains en Rhodésie du Nord

The Kalela Dance. Aspects of Social Relationships among Urban Africans in Northern Rhodesia

\section{J. Clyde Mitchell}

Traduction : Michel Agier et Stéphane Nahrath

James Clyde Mitchell est né le 21 juin 1918 à Durban, en Afrique du Sud. Il étudie la sociologie dans cette même ville, à l'Université du Natal, où il obtient le titre de Bachelor of Arts en Sciences sociales en 1942. Après avoir été volontaire de la Royal Air Force pendant la Seconde Guerre mondiale, il est intégré en 1946, comme chercheur assistant, au Rhodes-Livingstone Institute (RLI), à Lusaka, en Rhodésie du Nord (actuelle Zambie), au moment où Max Gluckman, Sud-Africain de Johannesburg, en est le directeur ${ }^{1}$. Clyde Mitchell mène jusqu'en 1949 une étude anthropologique au Malawi du sud. La monographie qu'il en tire étudie les formes traditionnelles et modernes de l'autorité politique et du conflit dans des villages Yao dans le contexte de l'administration coloniale. Elle lui permet d'obtenir son Ph.D. à Oxford en 1950 et sera publiée, sous une forme révisée, six ans plus tard, par les Presses de l'Université de Manchester'. Il devient en 1950 Senior Sociologist puis, en 1951, directeur du Rhodes-Livingstone Institute (il le restera jusqu'en 1955). C'est durant cette période (1950-1951) qu'il dirige une recherche collective sur la population africaine du Copperbelt, dans laquelle prend place son étude particulière sur la danse du kalela, qui reste son ceuvre la plus marquante. En 1955, il participe à la fondation du Département de Sociologie de l'Université de Rhodésie et Nyasaland, à Salisbury - l'actuelle Université du Zimbabwe à Harare où il est professeur d'Études africaines jusqu'en 1964. Il intègre ensuite l'Université de Manchester en 1966 comme professeur de Sociologie urbaine, poste qu'il occupe jusqu'en 1973. Le reste de sa carrière se déroule au Nuffield College, Oxford, d'abord comme Fellow de 1973 à 1985, puis comme Emeritus Fellow de 1985 à 1995. Tout en vivant et travaillant en Grande-Bretagne, il garde des contacts réguliers avec parents, amis et collègues en Afrique du Sud, où il se rend régulièrement. Clyde Mitchell est décédéle 15 novembre 1995. 
2 Le nom de Clyde Mitchell est d'abord associé au Copperbelt - les mines de cuivre de l'Afrique australe. Cela grâce à l'étude pionnière de la danse de kalela, menée à Luanshya, ville minière fondée en 1920, mais aussi grâce à plusieurs autres études sur les migrations de travail, l'urbanisation, les hiérarchies socio-professionnelles, la famille et l'ethnicité menées dans ce même cadre. Il fut ainsi, selon les termes d'un autre chercheur de l'école de Manchester, Bruce Kapferer', "la figure majeure des recherches sur l'urbanisation africaine ». Par la suite, Clyde Mitchell a activement participé à la réflexion sur la définition et les usages possibles de la network analysis ${ }^{4}$ et surtout à la systématisation de la perspective situationnelle. Il a pris position, dans les débats sociologiques, contre l'«objectivisme» et le "scientisme» des années cinquante-soixante, et se sentait proche de l'interactionnisme symbolique. Mais son inspiration la plus marquante se situe dans la tradition anthropologique et ethnographique. Ses références à Evans-Pritchard sont nombreuses et il y chercha notamment l'origine plus lointaine de l'approche situationnelle ${ }^{5}$. Ainsi, il recourt à l'idée de " sélection situationnelle » d'Evans-Pritchard pour formuler le point de vue selon lequel la "situation est définie " par le sens qu'y engagent les acteurs: Evans-Pritchard l'intéresse lorsqu'il parle de "la plasticité des croyances en fonction des situations », ce qui permet à Mitchell de dire que, si la culture peut exister comme " un vaste entrepôt de significations construit au long du temps et auquel les gens peuvent ajouter ou retirer des éléments ", c'est la situation qui fait le choix entre des éléments divers, voire contradictoires, de ce stock. L'approche situationnelle débouche ainsi sur une conception de la culture qui rompt avec l'immobilisme des analyses structurales ${ }^{6}$. L'autre inspiration marquante et plus directe de Mitchell se trouve dans les recherches effectuées dans le même Institut par Max Gluckman, aîné et maître reconnu de l'ensemble des chercheurs de l'école de Manchester. La recherche de Gluckman sur la vie sociale et politique du Zululand sous domination blanche présentée à partir de la description d'un événement particulier (les comportements des participants à l'inauguration d'un pont, dans la même région, par un fonctionnaire blanc) a représenté le point de départ de Mitchell pour élaborer l'approche de Kalela Dance et pour développer plus tard sa perspective situationnelle.

3 Kalela Dance a fait l'objet d'une première version en 1951, rédigée à partir d'enquêtes réalisées en 1950-1951. Présenté dans plusieurs séminaires au Rhodes-Livingstone Institute et à l'Université de Manchester, le texte a été repris entre 1953 et 19557. La publication définitive date de 1956 (The Kalela Dance, Rhodes-Livingstone Institute Paper, 27, Manchester, Manchester University Press, 1956). Considéré par Fredrik Barth comme une étude exemplaire des processus de différenciation et de définition des frontières ethniques en villes, commenté par Ulf Hannerz comme un modèle de l'analyse de situation', Kalela Dance a représenté au milieu des années cinquante une innovation de méthode et de contenu pour l'ethnologie. Norman Long a récemment souligné ${ }^{10}$ l'intérêt de la ligne d'analyse développée dans Kalela Dance: celle-ci, "en mettant en évidence la nature situationnelle des identités ethniques changeantes et la discontinuité des systèmes tribaux ruraux et urbains, a remis en cause les notions existantes de détribalisation et les modèles dualistes simples opposant les phénomènes urbains et ruraux ».

4 Ajoutons que ce petit ouvrage (une cinquantaine de pages denses dans la version originale anglaise) offre une contribution aux débats en cours aujourd'hui sur les échelles d'enquête et d'analyse. En effet, l'objectif de Kalela Dance, c'est bien la compréhension des relations raciales, ethniques et de classe dans la société globale-locale du Copperbelt. Mais, pour l'atteindre, Mitchell suit la procédure suivante:

1) description-question (la danse, réalisée par un même groupe ethnique et qui met en scène des caricatures de Blanc, est-elle d'imitation-dérision raciale ou de compétition inter-ethnique ?) ; 2) contextualisation partielle et raisonnée (relations raciales, tribalisme, régionalisme, travail et urbanisation en Rhodésie du Nord) ; 3) retour à la situation et conclusion déportée globale-locale et 
théorique (la danse de kalela exprime une identité ethnique en tant que forme de catégorisation sociale urbaine dans l'univers de relations intra-africain du Copperbelt).

Ainsi, au-delà de l'intérêt pour les données elles-mêmes, l'apport actuel de Kalela Dance réside-t-il dans le caractère transposable de son apport méthodologique.

MICHEL AGIER et STÉPHANE NAHRATH

6 Kalela est le nom d'une danse populaire «tribale » du Copperbelt en Rhodésie du Nord. Certains éléments curieux ont attiré mon attention alors que j'étais engagé dans une recherche de terrain où je l'avais utilisée comme fil conducteur d'une enquête générale sur le tribalisme et sur quelques autres caractères des relations sociales entre Africains dans les villes de la Rhodésie du Nord.

7 En présentant ici ce matériau et son analyse, j'ai essayé de suivre la méthode utilisée par Gluckman dans son Analysis of a Social Situation in Modem Zululand ${ }^{11}$. Gluckman commence son article par une description de la cérémonie d'inauguration d'un nouveau pont par le commissaire en chef local. Il isole les éléments importants de la cérémonie et fait ensuite remonter chacun de ces éléments jusqu'à la société dans son ensemble afin d'expliquer leur signification dans la cérémonie qu'il a décrite. En observant les comportements dans la cérémonie, Gluckman est conduit à une analyse historique et sociologique de la structure sociale du Zululand moderne dans sa totalité.

Dans ce texte j'essaie d'employer les mêmes techniques générales. Je commence par une description de la danse du kalela puis je mets en rapport les caractères dominants de la danse avec le système de relations entre Africains au Copperbelt. Pour établir ce rapport, je dois prendre en compte, jusqu'à un certain point, le système général des relations entre Noirs et Blancs en Rhodésie du Nord. Ainsi, en partant d'une situation sociale spécifique au sein du Copperbelt, c'est toute la construction sociale du Territoire qui est comprise dans l'analyse. C'est seulement lorsque ce processus a été mené à son terme que nous pouvons retourner à la danse et évaluer pleinement sa signification.

\section{Kalela}

9 La pratique de la danse tribale est devenue un trait de la vie urbaine à travers toute l'Afrique australe. Dans le Witwatersrand, les danses militaires collectives des Nguni sont devenues un spectacle pour touristes lors de la visite de Johannesburg. Ce type de danse est aussi devenu une sorte de loisir organisé durant lequel des équipes de danseurs s'opposent chaque semaine ${ }^{12}$. Au Copperbelt, la pratique de la danse "tribale » n'en est pas moins une caractéristique de la vie africaine. À la différence des somptueuses danses militaires empanachées et cadencées des Nguni, les danses tribales du Copperbelt sont assez discrètes et, en comparaison avec les danses du Sud, presque prosaïques. Pourtant, chaque township, cité ou quartier africain a ses endroits où des équipes de danseurs de différentes tribus se donnent en spectacle, chaque dimanche après-midi et les jours fériés.

Les Ngonde, les gens originaires du Kasai, les Nsenga, les Cewa, les Ngoni, et beaucoup d'autres tribus ont leurs propres danses distinctives. Mais la danse la plus populaire entre toutes est le kalela ${ }^{13}$, qui est dansé dans tout le Copperbelt par les gens de la province du 
nord de la Rhodésie du Nord. Au cours de l'année 1951, j'ai pu observer plusieurs danses kalela exécutées par une équipe Bisa dans la cité minière de Luanshya. J'ai également pu réunir des éléments d'information sur l'origine sociale des danseurs ${ }^{14}$. La description de la danse et des danseurs se base donc sur l'exemple de cette équipe.

L'équipe était constituée de dix-neuf jeunes hommes. Le costume du danseur de base était constitué d'un pantalon gris bien repassé, d'un gilet soigné et de chaussures bien cirées. Certains danseurs portaient un mouchoir blanc dans leur main droite. Leurs cheveux étaient soigneusement peignés avec une raie bien marquée. En résumé, c'étaient des jeunes gens habillés élégamment dans le style européen. L'équipe dansait au son de trois gros tambours fabriqués à partir de barils d'essence de 44 gallons recouverts d'une peau de vache. Deux batteurs frappaient les tambours avec des bâtons recourbés en forme de banane longs de deux pieds environ. Ce bruit de tambour, qui était assourdissant dans l'arène de danse même, pouvait être entendu à plusieurs miles de distance aux alentours. Les tambours étaient suspendus à un mât se trouvant au centre d'une clôture fermée sur la place et les danseurs circulaient autour d'eux en file indienne. La danse consistait en petits pas traînants effectués avec le haut du corps penché en avant et accompagnés de légers mouvements de rotations des hanches. De temps à autre le leader de la troupe ponctuait la cadence des percussions par des coups aigus de sifflet d'arbitre de football, après quoi les danseurs se tournaient à l'unisson vers les tambours. Durant une partie de la danse, les tambours restaient silencieux pendant que les danseurs chantaient.

\section{Les équipes de danse}

12 Chaque équipe de danse est organisée de la même manière. La composition de celle dont je viens de parler est la suivante. À sa tête, on trouve un " roi $^{15}$ ", élu par les membres de l'équipe de danse pour servir d'organisateur et d'administrateur général. Il est également leur trésorier : les membres lui paient leur cotisation lorsqu'ils vont en équipe participer à une compétition au cours de laquelle ils rencontrent d'autres danseurs de kalela dans une autre ville du Copperbelt, ou toutes les fois qu'ils célèbrent une fête. Lorsque j'ai observé la danse, le "roi » était vêtu de manière sensiblement différente des autres danseurs : il portait un complet sombre, un faux col et une cravate, un chapeau et une paire de lunettes de soleil à montures blanches. Après un certain temps, il interrompit la danse pour serrer la main de chaque danseur à la manière d'une célébrité qui irait à la rencontre des équipes lors d'un match de football.

Le meneur de la danse s'appelait Luke Mulumba et avait succédé à son frère à cette position en 1948. C'est en fait lui qui mène la danse tandis que le « roi » n'y prend aucune part active. Le meneur invente les pas de danse et compose les paroles des chants, autant d'éléments qui sont particulièrement importants. Un "docteur " vêtu d'une blouse blanche de chirurgien frappée d'une croix rouge sur le devant était également présent. Sa tâche était d'encourager les danseurs. Une " sœur-infirmière », la seule femme du groupe, était habillée en blanc et circulait entre les danseurs avec un miroir et un mouchoir à la main de façon que chacun puisse vérifier de près s'il était bien soigné et propre. Elle essuyait aussi la sueur sur le visage des danseurs pendant qu'ils dansaient. Elle est la sœur de Luke et est mariée avec le « roi ». Le reste de l'équipe est composé de danseurs et de batteurs. [...]

Cette équipe est manifestement composée d'hommes en majorité originaires de la chefferie bisa du Chef Matipa. Luke Mulumba, le meneur, qui de fait domine l'équipe, est 
un fils de la sœur de Matipa et il est évident qu'il a attiré autour de lui un certain nombre de sujets du frère de sa mère. Ses chants font l'éloge du Chef Matipa et par conséquent, indirectement, le sien.

15 Mais il y a également cinq hommes originaires d'une chefferie bisa voisine qui est sous l'autorité du Chef Chiwa ${ }^{16}$. Ces cinq hommes sont facilement acceptés au sein de l'équipe du fait que, comme nous allons le voir par la suite, dans le cadre du Copperbelt, l'équipe de Luke Mulumba est représentative de tous les Bisa.

16 Les Ng'umbo placés sous l'autorité du Chef Mwewa et les Aushi de la région du Chef Milambo avaient aussi des équipes de kalela, et il existait une équipe Bisa composite de kalela formée de membres de toutes les chefferies reconnaissant la suprématie du Chef Kopa. L'équipe de Mulumba a été formée avec pour objectif de faire la louange du Chef Matipa et s'est séparée de l'autre équipe Bisa pour pouvoir le faire. Cependant, en public [...] ils évoquent le symbole du chef suprême afin d'exprimer leur unité contre toutes les autres tribus, en même temps qu'ils expriment le prestige du Chef Kopa en termes singulièrement modernes ${ }^{17}$. Il est donc tout à fait possible pour les Bisa n'appartenant pas à la chefferie du Chef Matipa de participer à cette danse. Les différences internes sont ignorées face à la situation multi-tribale urbaine.

17 L'origine tribale commune de l'équipe mise à part, il y a d'autres régularités significatives. Personne au sein de l'équipe n'a plus de trente ans. La plupart ont moins de vingt-cinq ans. Il est vrai qu'au Copperbelt les hommes ont tendance à être choisis parmi les groupes d'âges les plus jeunes, mais l'équipe de Mulumba est encore plus jeune que la moyenne de la population du Copperbelt ${ }^{18}$. D'autre part, le « roi » est âgé de quarante et un an.

18 Une autre régularité marquante est que tous les danseurs vivent dans les logements pour célibataires. Trois des danseurs sont mariés mais leurs femmes sont restées à la campagne. Tous les autres sont soit célibataires, soit divorcés. Le « roi» toutefois est marié et sa femme, qui est la sœur de Luke Mulumba, est la «sœur-infirmière » de l'équipe.

19 Le fait que tous les danseurs chrétiens soient catholiques n'est pas en soi significatif dans la mesure où la mission catholique est la seule à opérer dans la région de Matipa. Mais il est intéressant ici de noter que le "roi », une fois de plus en contraste par rapport aux danseurs, est un adhérent de l'église Watchtower ${ }^{19}$.

20 Plus intéressant est le fait qu'aucun des danseurs n'occupe un emploi de « col blanc » ni de technicien, fait qui sera important à la lumière de la discussion qui suit.

\section{Le chant}

21 Une simple promenade à travers la cité minière de Luanshya un dimanche après-midi suffit pour se rendre compte de l'écrasante popularité, parmi les spectateurs africains, du kalela par rapport à toutes les autres danses tribales. Alors qu'il peut n'y avoir qu'une poignée de gens regardant les autres danses, l'arène du kalela est bondée de spectateurs visiblement en train de se divertir. Il y a plusieurs raisons à cette popularité. Les tambours sont spectaculaires et les danseurs bien habillés, mais je pense que la raison principale de cette attirance se trouve, de loin, dans les chants de l'équipe. Il est sans doute significatif que ces chants soient chantés en langue Bemba, langue qui est largement parlée au Copperbelt. Comme les danseurs utilisent la lingua franca urbaine, les spectateurs comprennent plus facilement leurs chants que ceux qui sont chantés par 
certains autres groupes tribaux dans une langue probablement compréhensible seulement par quelques étrangers.

$$
\text { un }
$$
mon point de vue, est que le kalela est essentiellement une danse tribale. Le kalela et ses chants mettent en avant l'unité des Bisa en opposition à toutes les autres tribus du Copperbelt. On pourrait naturellement s'attendre, dans le cas d'une danse tribale de cette sorte, à ce que quelques signes distinctifs tribaux soient arborés. Il n'est pas difficile de voir, dans une phalange de guerriers zoulous parés d'un costume traditionnel et brandissant sagaies et boucliers, une démonstration manifeste et même agressive de l'unité tribale. Mais les danseurs du kalela sont, eux, parés des vêtements européens les plus chics et il n'est pas possible de distinguer une équipe de kalela Bemba ou Aushi d'une équipe Bisa. L'élégance des danseurs de kalela est un thème récurrent, très fortement mis en relief ${ }^{20}$. Les chants ne sont pas non plus des récits des exploits d'un héros de la culture Bisa. Quelques références au beau pays de Matipa mises à part, aucune mention n'est faite aux plantations et aux récoltes, à la construction des cases, à la pêche et à la chasse ou à d'autres activités rurales que l'on pourrait associer à une espèce d'Arcadie tribale. Au lieu de cela, les chants traitent de situations familières de la vie au Copperbelt, les personnages sont des personnages familiers et les lieux sont situés dans les quartiers des cités. La langue des chants est le Bemba du Copperbelt au sein duquel abondent les mots et les phrases en anglais et en «kitchen kaffir». Les chants sont des compositions urbaines, pour divertir les gens de la ville et ils traitent d'événements et de lieux communs qui leur sont familiers.

En d'autres termes, nous sommes en présence d'un paradoxe apparent. La danse est clairement une danse de caractère tribal, dans laquelle les différences tribales sont rehaussées, mais la langue, l'expression des chants et l'habillement des danseurs puisent leurs références dans un mode de vie urbain qui tend à noyer les différences tribales. 

cadre social.

\section{L'origine de la danse}

Mes informateurs m'ont dit que la danse appelée kalela était autrefois connue sous le nom de mbeni. Ils m'ont également dit que le kalela avait commencé autour de 1930 sur l'île Chishi du lac Bangweulu par un homme appelé Kalulu. Les habitants de cette île appartiennent à la tribu des Ng'umbo. En 1939, Kalulu s'engagea dans le Régiment de Rhodésie du Nord et eut la permission d'emporter avec lui ses tambours. Il put ainsi, lorsque l'occasion le permettait, poursuivre sa pratique de la danse. Il forma un groupe de danseurs sous sa propre direction. Un certain Million était le meneur des danses sur l'île de Chishi quand Kalulu était à l'armée. Kalulu, licencié de l'armée en 1946, rebaptisa la danse du nom de luwelela ${ }^{21}$. Nous ne savons pas qui l'introduisit au Copperbelt ${ }^{22}$ mais, dans la mesure où le mouvement de population en direction du Copperbelt s'est accru après la guerre de 1939-1945, il est à peu près certain qu'elle a été apportée là par quelques membres de la tribu des Ng'umbo en provenance de l'île Chishi. À ce que je comprends, elle arriva d'abord aux mines de cuivre de Roan Antelope en 1945, d'où elle se diffusa dans le reste du Copperbelt, et dans la cité de Luanshya en particulier en 1948. Là elle fut appelée kalela - une danse d'orgueil.

\section{Mbeni}

Puisque le kalela vient du mbeni, il convient de remonter aux origines du mbeni pour retrouver ses racines. Malheureusement, il semble qu'il n'y ait que peu d'enregistrements disponibles de la danse mbeni. Durant mon travail de terrain au Nyasaland ${ }^{23}$, j'ai pu observer une danse mbeni lors d'une cérémonie d'initiation de jeunes gens. Les exécutants étaient un groupe de jeunes quelque peu sales et négligés qui circulaient sans énergie dans l'arène de danse en suivant une imposante grosse caisse de fabrication artisanale. L'un de mes plus vieux informateurs me dit que ce spectacle n'avait que peu de ressemblance avec les danses beni qui étaient exécutées au Zomba dans les années vingt. Il me raconta que le mot beni, qui sert à nommer cette danse dans le Nyasaland, est en fait une déformation du mot anglais «band». Cette explication de l'origine du mot semble raisonnable à la lumière de la description de la danse en elle-même dont la caractéristique essentielle, à ce que nous pouvions voir, était de constituer une sorte de simulacre de troupe militaire ${ }^{24}$. Comme me le disait mon informateur : «C'était une danse propre parce que tout le monde portait de beaux habits. Les gens sales n'étaient pas autorisés à danser. Chaque fois qu'ils étaient appelés, ils prenaient leurs tambours et venaient vêtus comme le Roi. Lorsqu'ils arrivaient dans la cour où la danse devait avoir lieu, ils paraissaient splendides. Les femmes aussi étaient très propres. Tous dansaient lentement et en douceur, les femmes d'un côté et les hommes de l'autre ; au lever du jour ils paraissaient aussi propres que s'ils n'avaient pas dansé du tout. »

31 La figure centrale de ces danses était apparemment une personne appelée «le Gouverneur ». Il se tenait généralement au centre de la cour, vêtu de manière resplendissante, décoré de médailles d'emprunt. Les danseurs tournaient lentement autour de lui emmenés par un batteur battant la mesure sur une grosse caisse d'imitation. 
Le reste des danseurs était aligné derrière lui en une parodie de défilé militaire. En premier, il y avait un major-général, suivi par un lieutenant-général ${ }^{25}$, un colonel, un lieutenant-colonel, un capitaine, des lieutenants, des sous-officiers et, finalement, les soldats. Il y avait également un adjudant. Les danseurs portaient les insignes en plomb conformes à leur grade. Ceux qui occupaient les rangs d'officiers fictifs portaient des casques et avaient des sifflets à lanières tressées, et quelques-uns portaient des ceinturons de tunique Sam Browne ${ }^{26}$.

Les relations au sein du groupe de danseurs étaient réglées selon les grades militaires fictifs. Les difficultés et les disputes entre membres du groupe étaient aplanies par celui qui était le supérieur immédiat en grade et, si aucun arrangement ne pouvait être trouvé, ce dernier en référait à ses supérieurs en suivant la hiérarchie jusqu'au "Gouverneur » lui-même.

Goodall, témoignant devant la commission Russell, soutenait l'idée que la danse mbeni avait surgi peu après la guerre de 1914-1918 ${ }^{27}$. Il mentionne l'existence de la danse en 1919 à Dar-es-Salaam. Les autorités s'y intéressèrent au moment des émeutes de 1935, bien qu'il soit difficile de déterminer quel rôle les groupes de danse mbeni y ont joué, s'ils en ont jamais vraiment joué un. Il est évident que les fonctionnaires du gouvernement soupçonnaient les danseurs mbeni d'être impliqués dans ces émeutes et il semble que, en l'absence de toute organisation stable à travers laquelle les leaders africains auraient pu communiquer l'information à la population, les leaders de la grève ont pu demander aux meneurs des danseurs mbeni d'être leurs porte-parole ${ }^{28}$.

Le point important qui ressort du témoignage devant la Commission était que la danse sous la forme que nous lui connaissons était pratiquée au Copperbelt en 1935. Jusqu'à quel point le mbeni devint suspect à la suite de la méfiance qu'il avait éveillée durant cette année, je ne puis le dire, mais il semble avoir disparu du Copperbelt ${ }^{29}$ avant de renaitre sous la forme du kalela.

\section{Prestige et « mode de vie européen »}

Quelle que soit la forme prise par les danses mbeni modernes ${ }^{30}$, il apparait très clairement que ces premières danses étaient une sorte de pantomime de la structure sociale de la communauté européenne locale. Mon informateur Yao me décrivait le mbeni à Zomba, alors que cette ville était, pendant les années vingt, essentiellement une ville de garnison. Le gouverneur et la milice incarnaient aux yeux des Africains une structure sociale formelle, dont les traits saillants étaient une hiérarchie rigide et une collection d'uniformes différents indiquant la position sociale de chacun. La pantomime de la structure sociale dans le mbeni représentait ainsi la structure sociale telle que les Africains la percevaient. Il faut tenir compte du fait que, dans les années vingt, les Africains n'étaient pas considérés par les Européens de Zomba comme leurs égaux et n'avaient donc l'occasion de se faire une idée du modèle des relations sociales au sein de la communauté locale qu'à travers les grades militaires ${ }^{31}$, ainsi qu'à travers les signes évidents des uniformes et des cérémonies publiques ${ }^{32}$. L'attrait pour la danse mbeni semble ainsi provenir du fait qu'elle représentait pour les Africains une sorte de participation indirecte aux relations sociales dont ils étaient normalement exclus. Une preuve saisissante que ce n'était pas seulement une réaction locale est fournie par Goodall qui raconte que les plus anciens danseurs de mbeni se blanchissent véritablement le visage $^{33}$. Cette tentative de franchir, par l'imagination, des barrières sociales 
insurmontables est une caractéristique particulière des mouvements nativistes ${ }^{34}$ tel que le culte du cargo. Mais il existe une différence certaine: il n'y a pas de preuve que les danseurs de mbeni aient jamais cru qu'en reproduisant les traits extérieurs de la culture à laquelle ils aspiraient, ils atteindraient automatiquement leurs désirs. Leur participation à la structure sociale "européenne » se faisait par procuration: l'aspiration n'était satisfaite qu'en parodie.

On pourrait arguer du fait que la danse fournissait un excellent moyen d'expression de l'hostilité à l'encontre du groupe dominant à travers l'usage de la satire et que c'est cela qui constituait la satisfaction principale pour les participants et les spectateurs. Je n'ai aucune preuve que c'était vraiment le cas. Mon informateur Yao ne l'a jamais suggéré et aujourd'hui il n'y a certainement dans la danse de kalela aucun signe d'une intention satirique à l'égard du comportement européen ${ }^{35}$.

Tout ce qui a été conservé du mbeni dans le kalela moderne c'est le port de vêtements européens ainsi que certains personnages types tels que le « roi », le «docteur», et la «sœur-infirmière ». Du fait que tous les Africains de Rhodésie du Nord portent de nos jours des vêtements européens, on peut s'attendre à ce que les danseurs ne fassent pas différemment. Mais le trait saillant des deux danses mbeni et kalela est la grande exagération donnée au caractère correct de l'habillement. Aux yeux de mon informateur, c'était là la caractéristique marquante de la danse. Dans sa description de la danse mganda, Jones précise : «Et ensuite arrivèrent les officiers vêtus d'habits européens, très chics, et brandissant leurs cannes à la manière d'un cavalier.» Il est particulièrement significatif que la mascotte du régiment dans la danse du mganda était « une de ces têtes en bronze utilisées comme publicité, je crois pour les cols de chemises Van Heusen, coiffées d'un chapeau en feutre ordinaire ${ }^{36} »$. Dans le kalela également il y a cette forte insistance sur la tenue impeccable. Les danseurs y font référence dans leur chant; la sœur-infirmière porte un miroir en faisant le tour des danseurs pour qu'ils puissent vérifier leur apparence ; un journaliste africain, écrivant un article sur la danse du kalela pour un journal local, se fait un point d'honneur à mentionner l'élégance de l'habillement 37.

Cette insistance sur l'élégance de l'habillement est un trait caractéristique général de la population urbaine africaine ${ }^{38}$. Wilson l'exprime de la manière suivante : «Les Africains de Broken Hill ne sont ni des vachers, ni des chevriers, ni des pêcheurs, ni des bûcherons, ils portent des habits ${ }^{39}$." Wilson voyait l'origine de cette préoccupation pour l'habillement dans le fait que les vêtements sont un des objets facilement accessibles de la richesse européenne qui leur donne une apparence immédiate de statut civilisé ${ }^{40}$. Il analysa d'autres indicateurs possibles du statut civilisé - le logement, l'outillage, le mobilier, la nourriture - mais conclut que, pour toute une série de raisons, ils étaient sans importance comparativement à l'habillement à Broken Hill en 1939-1940 ${ }^{41}$. Il remarqua très clairement que «les Africains désirent, bien que ne le pouvant pas, acquérir le respect et partager le statut civilisé ainsi que la nouvelle richesse des Européens dont la supériorité sociale se trouve toujours sous leurs yeux ${ }^{42}$ ».

Les observations de Wilson, valables pour Broken Hill en 1939-1940, sont également valables pour le Copperbelt moderne. Les Européens se trouvent en position de supériorité sociale et les Africains aspirent à la civilisation qui caractérise, avec ses petits avantages propres, le groupe socialement supérieur ${ }^{43}$. Le mode de vie civilisé définit ainsi une échelle à partir de laquelle peut être mesuré le prestige des Africains des régions urbaines (ainsi que d'une proportion toujours plus grande de régions rurales). Au sommet 
de l'échelle se trouvent les techniciens, les « cols blancs » et les négociants prospères qui tous sont habillés avec un soin méticuleux, possèdent du mobilier européen dans leurs maisons, parlent anglais entre eux, lisent le journal local destiné au public européen, mangent de la nourriture de type européen, préfèrent la musique occidentale à la musique traditionnelle, choisissent des bières en bouteilles de préférence à la bière brassée à l'ancienne. Au bas de l'échelle se trouvent les travailleurs non spécialisés de toutes sortes dont le niveau de vie ne diffère que très peu de celui des villageois ruraux et qui ne possèdent pas de mobilier, mangent de la nourriture traditionnelle, ne savent pas du tout parler anglais et ne sont pas instruits. Entre les deux, on trouve les employés subalternes, les contremaîtres et les ouvriers qualifiés, avec des différences considérables quant au niveau qu'ils peuvent atteindre de ce qu'ils croient être « un mode de vie civilisé ».

Une étude de la hiérarchie professionnelle confirme cette conception du prestige au sein de la communauté urbaine africain $\mathrm{e}^{44}$. Les personnes interrogées étaient invitées à classer 31 professions sur une échelle de prestige en cinq points. Par la suite, lorsque les données furent converties en un classement simplifié, les cadres étaient placés en tête, suivis par les autres cols blancs, ensuite les ouvriers qualifiés et les contremaîtres et, finalement, les travailleurs non qualifiés. Les réponses à une question ouverte finale permettaient très clairement de voir que les professions qui étaient normalement celles des Européens, mais que certains Africains exerçaient, se voyaient accorder un prestige élevé et que, en règle générale, celles qui exigeaient les diplômes scolaires les plus élevés étaient classées au plus haut de la hiérarchie. Ce constat valait également pour un groupe d'étudiants qui suivaient une formation d'artisans.

41 L'usage africain du mode de vie européen comme étalon de mesure du prestige peut donc être considéré comme un genre de comportement avec groupe de référence (reference group behaviour) ${ }^{45}$. Les danseurs de mbeni recouraient à un type très direct de comportement à groupe de référence. Ils copiaient les symboles de prestige les plus manifestes et les plus visibles; Le lien entre le mbeni et le kalela est maintenu grâce à un commun usage de l'habillement comme un tel symbole. Les danseurs de kalela n'utilisent plus l'uniforme militaire, mais les vêtements élégants des hommes d'affaire et des cadres européens : les Africains en sont généralement arrivés à accepter les modèles de ces gens comme ceux auxquels ils aspirent eux-mêmes. Les symboles directs et manifestes ouvrent ainsi la voie à l'idéal, moins tangible mais néanmoins réel, du mode de vie civilisé. Le mécanisme est le même mais les symboles sont aujourd'hui différents.

Il est significatif qu'aucun des danseurs de kalela n'occupe un poste de cadre ou autre "col-blanc». Trois d'entre eux sont tailleurs: les autres sont des travailleurs non qualifiés de différentes sortes. Pour une équipe de danseurs dont les membres exercent des professions se trouvant à l'extrémité la plus basse de l'échelle de prestige, le fait de porter d'élégants vêtements de style européen est particulièrement important. Ceux qui en vertu de leur position dans la communauté ne disposent dans la vie de tous les jours que de peu de prestige, se réapproprient le dimanche les symboles et les marques extérieures du rang social et les affichent face aux spectateurs admiratifs autour de l'arène de danse. Le mode de vie européen fait désormais tellement partie de la vie dans les aires urbaines, qu'il n'est plus directement associé à la présence des Européens euxmêmes. Les danseurs de kalela ne recherchent pas une participation par procuration à la société européenne, mais une participation indirecte aux couches supérieures de la société africaine, dont ils sont exclus par leur manque de qualification. Le système de 
prestige dans les régions urbaines utilise ainsi «la civilisation» ou "le mode de vie européen » comme modèle ou comme échelle de prestige. Pour inspirer le respect dans un tel système, l'Africain doit avoir été scolarisé, occuper un poste offrant un prestige élevé, et gagner un salaire suffisant pour lui permettre d'acquérir des vêtements et d'autres symboles de prestige. La population urbaine africaine est stratifiée selon les critères de cette échelle. [...]

Plusieurs groupes recrutent leurs membres parmi les Africains placés à des niveaux particuliers du système de prestige. Quelques-uns de ces groupes comme l'équipe de kalela recrutent sur une base à la fois tribale et de classe : ses membres sont tous des Bisa situés aux rangs inférieurs de l'échelle de prestige. Il est possible, bien que je n'aie aucune preuve pour l'affirmer, que certains cultes religieux recrutent leurs membres dans toutes les tribus et parmi les seules couches inférieures du système de prestige.

Le fait que des membres de certains groupes constitués tels que les équipes de kalela soient recrutés à des niveaux particuliers du système de prestige est intéressant, et nous essayons de comprendre pourquoi il en est ainsi. Mais la position dans le système de prestige n'est pas la raison d'être ${ }^{46}$ spécifique de ces groupes: ils existent pour servir d'autres intérêts. Pour autant que je le sache, les Africains des couches inférieures du système de classement ne se sont jamais organisés en opposition à ceux du sommet de la hiérarchie. Occasionnellement toutefois, quelques groupes ont surgi avec l'objectif de pousser plus loin la défense de leurs propres intérêts vis-à-vis ${ }^{47}$ des Européens. On peut en voir un exemple au travers des sociétés de «bienfaisance » qui recrutaient leurs membres au sein de l'« intelligentsia ${ }^{48}$ ", indépendamment de leur origine tribale. Ces sociétés, bien que composées principalement d'Africains des niveaux supérieurs du système de prestige, étaient formées en vue d'améliorer les conditions de vie de tous les Africains vivant en ville sans se soucier de leur appartenance ni tribale ni de classe. Il était inévitable qu'elles finissent par adopter un point de vue politique. En temps utile, elles fusionnèrent pour former l'African National Congress (ANC) qui recrutait dans toutes les couches de la société et toutes les tribus.

Le fait essentiel est que les Africains considérés comme un tout représentent une catégorie politique majeure et les Européens une autre. Dans cette situation, les « cols blancs » sont appelés à devenir les leaders politiques africains parce qu'ils savent parler l'anglais, présenter leurs doléances et faire part de leurs revendications en termes aisément compréhensibles pour les Européens. Mais la catégorie des "cols blancs» représente ici les Africains dans leur totalité et ne constitue pas une catégorie politique opposée aux travailleurs manuels ${ }^{49}$. Madame McCulloch remarque qu'à Livingstone « il y a une lutte pour le leadership dans la ville entre l'élite Lozi et un certain nombre d'"étrangers" qui sont des personnes de choix en termes de richesse, d'éducation et de profession $^{50}$ ». Proportionnellement il y avait plus d'ouvriers qualifiés parmi les " étrangers ${ }^{51}$ » que parmi les Lozi, mais également, et de loin, moins de travailleurs manuels non qualifiés parmi les Lozi et les «étrangers " pris ensemble que parmi tous les autres groupes ethniques ${ }^{52}$. En d'autres termes, la lutte pour le pouvoir politique ne se déroulait pas entre travailleurs qualifiés et non qualifiés ou entre travailleurs manuels et non manuels, mais plutôt entre de larges groupes ethniques à l'intérieur de la même strate socio-économique générale.

Il apparaît ainsi que les Africains du Copperbelt comme classe politique ne sont pas encore divisés par leurs appartenances tribales, ni par leurs appartenances socioéconomiques. Les relations sociales quotidiennes entre Africains dans le Copperbelt sont 
toutefois affectées à la fois par les appartenances tribales et les appartenances de classes socio-économiques, et les preuves dont je dispose suggèrent qu'actuellement les appartenances tribales sont de loin les plus importantes.

\section{Le tribalisme dans les villes}

47 L'habillement distinctif "moderne» des danseurs de kalela peut ainsi être imputé à l'importance du «mode de vie européen » et au rôle qu'il joue dans la stratification de la population africaine du Copperbelt. Les danseurs de l'équipe de Luke Mulumba provenaient, il faut le rappeler, d'une strate relativement basse du système de classement et, par le biais d'une sorte de mobilité ascendante fictive, ils étaient particulièrement fiers d'être capables de se parer de pantalons serrés, de gilets d'une propreté irréprochable et de chaussures bien cirées, qui leur allaient à merveille.

Nous avons aussi vu que la composition de l'équipe ne se faisait pas au hasard au sein des strates inférieures mais de manière exclusive parmi la tribu Bisa. L'équipe a en fait été formée dans le but de chanter les louanges du peuple Bisa en général, et elle remplissait aussi bien ce rôle que celui consistant à le distinguer des autres tribus du Copperbelt. On ne peut apprécier pleinement ce second élément de la danse du kalela qu'après avoir examiné la part que le tribalisme joue dans les interactions sociales entre Africains dans les zones urbaines.

Même à un niveau superficiel d'observation, l'importance du tribalisme dans les relations sociales quotidiennes au Copperbelt est évidente. La manifestation la plus claire en est naturellement les luttes tribales qui se produisent de temps en temps. Spearpoint raconte par exemple comment un homme originaire de la région de Kasai au Congo belge était entré en collision, avec sa bicyclette, avec deux Bemba et comment les membres des deux groupes s'étaient rapidement ligués avec des membres de leurs tribus respectives et avaient commencé à se battre ${ }^{53}$. Les rixes tribales ne sont pas aussi fréquentes au Copperbelt, mais on peut observer l'opposition entre tribus dans beaucoup d'autres situations. Les Tumbuka par exemple menaçaient en 1952 de se retirer de l'organisation de l'Église Libre du Copperbelt parce que les offices religieux étaient prêchés en langue bemba ; les Bisa ont à plusieurs reprises revendiqué devant le commissaire de district à Luanshya le droit d'avoir un assesseur bisa sur le banc de la cour de justice de la ville ${ }^{54}$. D. Chansa, un assistant de recherche de l'équipe du Rhodes-Livingstone Institute, rapporte dans une étude non publiée sur les coutumes des buveurs de bière le fait que $88 \%$ des 130 hommes de son échantillon déclaraient choisir leurs compagnons de boissons parmi les membres de leur tribu. À Broken Hill en 1940, Wilson découvrit « des groupes de commensaux dont la constitution était marquée tribalement, sans qu'elle le soit de manière exclusive ${ }^{55} \%$.

En Rhodésie du Sud, l'unité tribale des citadins africains s'exprime à travers les associations tribales de funérailles ${ }^{56}$. Les membres de ces sociétés paient une contribution d'environ

2 shillings 6 pences et ont droit en retour à une assistance financière et sociale en cas de deuil, ainsi qu'à certains avantages s'ils venaient à se trouver dans le dénuement. Il est très difficile d'estimer jusqu'à quel point ces associations de funérailles se retrouvent dans toutes les tribus que comptent les villes de Rhodésie du Sud. Dans son rapport annuel de fin d'année, daté de juin 1955, le directeur de l'administration indigène de Salisbury déclare que quinze associations de funérailles ont déposé leurs statuts auprès 
de lui pour sa propre information ${ }^{57}$. Combien d'autres ne l'avaient pas fait, nous ne le savons pas. Il y a certainement plus de quinze tribus présentes à Salisbury. En Rhodésie du Nord, il semble qu'il y ait, par contraste, beaucoup moins de sociétés tribales actives, excepté à Livingstone ${ }^{58}$. Seuls les Lozi paraissent avoir conservé une association tribale. Une association appelée "Les fils du Barotseland "semblait fonctionner avec succès au Copperbelt en 1951-1952. En 1954, le journal pour les employés africains, de la Compagnie Rhokana, Luntandanya, rapportait que la Société nationale des Barotse avait donné un concert à Nkana ${ }^{59}$. D. Chansa, dans son étude sur les habitudes des buveurs de bière, avait découvert à Lusaka des clubs de buveurs organisés sur des critères ethniques. Une société appelée « Cobra Drinking Company » avait été formée par un groupe de Ngoni lettrés. Ils dépensaient tous les fins de semaine leurs cotisations de club à boire des bières. Un club de buveurs kaonde ayant le même objectif était dirigé par un « roi » ainsi que par d'autres titulaires de fonctions officielles un peu à la manière d'une équipe de kalela. D'autres sociétés tribales ont certainement existé dans le passé et il s'en forme de nouvelles constamment. En août 1954 par exemple, l'African Roan Antelope rapportait que les Nyakyusa ont bu ensemble au coucher du soleil, et qu' « ils sont maintenant unis dans une compréhension mutuelle ». En septembre 1954 les Nyakyusa de Kitwe formèrent une société tribale. Mais, si, en Rhodésie du Nord, les sociétés tribales ont conservé un caractère intermittent, apparaissant et disparaissant soudainement, elles semblent, en Rhodésie du Sud, être devenues une partie intégrante de la structure sociale urbaine. [...]

L'élément qui ressort ici est l'exacerbation des sentiments tribaux par les situations sociales particulières qui se sont développées dans les villes nouvellement établies. L'arrière-pays où peut se recruter la main-d'œuvre des mines de cuivre est vaste. Pour la seule Rhodésie du Nord, on a dénombré quelque soixante-quinze groupes "tribaux » différents. Si l'on incluait ceux des régions proches de l'Angola, du Congo, du Tanganyika, du Nyasaland, du Mozambique, de la Rhodésie du Sud et du Bechuanaland, le nombre des groupes tribaux dont les travailleurs du Copperbelt pourraient provenir se monterait à quelques centaines. Mais le Copperbelt recrute sa main-d'œuvre d'une manière prédominante dans certaines régions ${ }^{60}$. Dans la cité minière de Luanshya, la distribution tribale des hommes adultes par groupes ethniques pris au sens large était en 1951 la suivante :

\begin{tabular}{|l|c|}
\hline Bemba, Aushi, Bisa, Lunda de l'Est, etc. & $34,2 \%$ \\
\hline Lamba, Lala, Swaka, Lima, etc. & $24,5 \%$ \\
\hline Nsenga, Chewa, Yao, Kunda, etc. & $16,5 \%$ \\
\hline Kaonde, Lunda de l'Ouest, Luchazi, etc. & $9,8 \%$ \\
\hline Lenje, Mazabuka, Tonga, Toka, etc. & $5,6 \%$ \\
\hline Ngoni & $4,0 \%$ \\
\hline Mambwe, Nyamwanga, Tumbuka & $2,6 \%$ \\
\hline Lozi & $2,2 \%$ \\
\hline Autres & $0,6 \%$ \\
\hline
\end{tabular}




\begin{tabular}{|l|l|}
\hline Total & $100,0 \%$ \\
\hline
\end{tabular}

Il n'existe pas de principe de distribution spatiale de ces tribus dans la cité. Il existe une longue liste d'attente pour les logements, de telle manière que, lorsqu'un logement se libère, il est attribué à la personne suivante sur la liste. Les tribus sont donc dispersées au hasard à l'intérieur de l'ensemble de la cité ${ }^{61}$. Il y a un va-et-vient considérable au sein de chaque section de la cité. Ceci est dû d'une part au fait que la main-d'œuvre africaine est encore largement migrante et d'autre part au fait que la plupart des logements sont loués au centre de gestion de la cité par les employeurs, de telle manière que le travailleur africain est obligé de changer de résidence à chaque fois qu'il change d'emploi. Il en résulte que la composition des sections au sein de la cité change constamment et qu'il y a peu de chances pour qu'une structure déterminée de communauté s'y développe.

C'est dans une situation comme celle-ci, où les voisins changent continuellement et où des individus de tribus différentes se trouvent soudainement mêlés les uns aux autres, que les traits distinctifs de l'autre deviennent apparents. Cette différence se manifeste de bien des manières, dont la plus importante, à n'en pas douter, est la langue. Mais l'habillement, les habitudes alimentaires, la musique, les danses, tout peut servir d'indicateur ou de signe distinctif de l'appartenance ethnique.

Ces moyens de classification par tribu permettent à un Africain vivant dans une cité ou un quartier, où les contacts sont nécessairement superficiels, de mettre tout autre Africain dans une catégorie fixe. De cette manière, il peut « définir la situation » et ainsi adopter un type de comportement approprié à l'égard des autres. [...]

\section{Le tribalisme et les relations catégorielles}

Cette tendance à réduire la large diversité des tribus en un nombre restreint de catégories fait partie d'un processus sociologique général qu'il est essentiel de saisir lorsque l'on veut comprendre les relations sociales parmi les Africains dans les zones urbaines.

Selon ce processus, les relations superficielles entre les gens sont déterminées par certaines grandes catégories au sein desquelles aucune distinction n'est reconnue. Nous pouvons l'examiner d'un peu plus près à la lumière du cas du Copperbelt. Là, la majorité de la population provient des tribus matrilinéaires des provinces du nord et du centre, tribus qui ont en commun, entre autres choses, un système de clan. A priori, nous pourrions déduire que, dans une situation urbaine où tant d'étrangers sont soudainement étroitement associés, le système de clan, qui est commun à un si grand nombre, fournit un mécanisme par lequel des liens entre voisins pourraient être forgés. En fait, mes données suggèrent qu'il n'en est rien. Une preuve de ceci est apparue clairement durant une excursion faite pendant un travail de terrain chez les Kaonde du district de Kasempa en Rhodésie du Nord ${ }^{62}$. Les Kaonde, comme beaucoup d'autres tribus de Rhodésie du Nord, sont organisés en clans matrilinéaires exogames. Parmi eux, la règle de l'exogamie est encore très forte: à Kasempa, pendant une courte excursion, W. Watson et J. van Velsen ne découvrirent aucun mariage entre des personnes ayant le même nom de clan. Lorsqu'ils rencontrèrent par hasard un tel cas, ils s'informèrent des circonstances dans lesquelles cela s'était produit. Le mariage avait été contracté au Copperbelt et le couple, alors qu'il se trouvait là-bas, ne s'était pas donné la peine de se demander mutuellement 
leurs noms de clan. Ils découvrirent qu'ils avaient commis un inceste de clan seulement lorsqu'ils revinrent dans leur région rurale d'origine où l'appartenance clanique est significative.

Un autre incident à Luanshya en 1951 corrobore l'idée selon laquelle la dimension clanique n'apparait pas comme une catégorie importante dans les relations sociales au Copperbelt. Une femme de la tribu Lenje, mariée à un Bisa, mourut brusquement. Son nom de clan matrilinéaire était chowa (champignon). Normalement, dans les régions régies par les principes tribaux, les devoirs funéraires auraient dû être accomplis par des membres du clan ayant avec le sien des relations à plaisanteries, c'est-à-dire le clan des chulu (la fourmilière). Comme je l'ai dit, les mêmes noms de clan apparaissent au sein d'une part importante des tribus prépondérantes au Copperbelt et nous aurions très bien pu nous attendre à ce que ce soit des membres du clan de la «fourmilière » au sein des Lenje, des Lamba, des Lala, des Swaka, des Lima, des Bemba, des Kaonde ou même des Bisa qui accomplissent les devoirs funéraires. En réalité, ce furent les Yao qui le firent. L'explication de cet état de fait est que la tribu Yao dans son ensemble entretenait une relation à plaisanterie avec les Bisa dans leur ensemble, qui est la tribu du mari de la défunte. Les Lenje, autant que je sache, n'entretiennent de relations à plaisanterie avec aucune autre tribu. En conséquence, dans cette situation urbaine où la tribu est la catégorie sociale significative, c'était la tribu à plaisanterie du mari qui se proposait pour l'accomplissement des devoirs funéraires.

L'expérience semble suggérer que les interactions ponctuelles entre Africains au Copperbelt sont essentiellement déterminées par l'appartenance à une tribu. Ce genre d'interactions est un aspect des relations catégorielles qui surviennent dans toute situation où les contacts doivent nécessairement être éphémères et transitoires. Le processus a été décrit en termes généraux par Shaler il y a quelques années : «Au début de toute relation, le rapport avec le semblable est inévitablement traité sur un mode catégoriel. L'autre est considéré comme membre d'un groupe, qui nous est indiqué par un petit nombre de signes commodes; au fur et à mesure que notre connaissance d'une personne particulière se développe, cette catégorie tend à se préciser. Ses limites sont repoussées jusqu'à ce qu'elles tombent ${ }^{63}$. » Hiller exprime la même idée : «La tendance à la catégorisation, parce qu'elle fournit un cadre de référence en matière de comportements avec les autres, qui va jusqu'à la possibilité de les exclure, permet une économie d'efforts dans les relations sociales. C'est spécialement le cas lorsque l'on a affaire à des étrangers. Classifier les gens revient implicitement à les connaître et à avoir un cadre de référence pré-arrangé en matière de relations ${ }^{64}$."

Que la catégorie de classement la plus importante dans les interactions sociales quotidiennes entre Africains au Copperbelt soit le tribalisme n'est pas surprenant. Il existe dans les villes un flot constant de nouveaux arrivants en provenance des différents districts ruraux d'où le Copperbelt tire sa main-d'œuvre. Ils ne sont pas immédiatement absorbés dans le système de prestige susceptible de fournir un principe alternatif d'interaction sociale. Au lieu de cela, leur propre différence ethnique, reçue comme allant de soi dans les régions rurales, prend immédiatement du relief face à la multiplicité des tribus avec lesquelles ils sont mis en relation. Leur spécificité ethnique se trouve ainsi à leurs yeux exagérée et elle devient le fondement de leur interaction avec tous les étrangers.

Une tribu dans les régions rurales est un groupe d'individus rassemblés par un système politique et social unique et qui partagent un certain nombre de croyances et de valeurs. 
Nous utilisons donc le mot «tribu » dans ce sens afin de désigner un groupe d'individus liés entre eux dans un système social particulier. Mais lorsque l'on parle de tribalisme dans un contexte urbain, on ne se réfère pas à un lien entre individus, déterminé selon une structure normée, c'est-à-dire une tribu, mais plutôt à une subdivision d'individus regroupés selon leur sentiment d'appartenance à certaines catégories, définies sur la base de critères ethniques. Le tribalisme au Copperbelt renvoie ainsi à des groupements constitués sur la base de larges différences culturelles. Il y a une tendance des Bemba et d'autres tribus des provinces du Nord à considérer les Chewa, les Nsenga, les Kunda et d'autres populations des provinces de l'Est, par exemple, comme des « Ngoni », et toutes les tribus du Nyasaland, bien qu'aussi différentes que les Tumbuka et les Lomwe, comme des « Nyasa ». De la même manière, les tribus des provinces de l'Est tendent à classer en bloc les Lungu, les Tabwa, les Lunda de l'Est, les Bemba, et d'autres tribus des provinces du Nord dans une seule catégorie - les « Bemba ».

Il est ainsi évident qu'il n'existe pas de corrélations nécessaires entre une structure tribale d'un côté et le tribalisme, au sens où j'emploie ce mot, de l'autre. La première est un système de relations sociales, l'autre est une catégorie d'interactions au sein d'un système plus large. Il semble que Harlow, dans l'un de ses rares articles concernant spécifiquement le tribalisme, n'ait pas réussi à faire cette distinction ${ }^{65}$. Il écrit par exemple : « Il existe beaucoup de preuves à l'appui de la thèse selon laquelle le tribalisme est, en Afrique, sur le point de disparaître ", et poursuit ensuite par la description des changements affectant la structure sociale tribale. Plus loin, il dit: "Sous la terrible pression des idées et des techniques occidentales, les Africains, dans beaucoup de régions, ont resserré leurs rangs afin de se préserver; et les seuls rangs qu'ils connaissent sont ceux de la tribu. D'où la réaffirmation agressive de l'identité et du prestige tribal. »

Comme preuve du fait que le tribalisme n'est pas en train de disparaître, Harlow cite l'exemple des Chagga qui ont récemment élu un chef suprême alors qu'ils n'en avaient auparavant jamais eu. Mais sa phrase contient un indice important : « Les Chagga se sont dotés d'un porte-parole qu'ils ont investi de prestige et d'autorité afin de parler avec les Européens ${ }^{66} . »$ Mon impression est que nous sommes en présence d'une population, autrefois liée de manière lâche, et qui est en train maintenant de s'unifier en opposition à un groupe extérieur d'Européens. L'identité Chagga est devenue une catégorie pertinente de l'interaction au sein d'un système social plus large que celui de la tribu. La structure interne de la tribu peut très bien subir de grands changements et le système tribal s'effondrer rapidement; mais il est toujours possible d'évoquer en un sens l'unité tribale en opposition à un groupe extérieur.

Mais le tribalisme des Chagga est un phénomène d'un autre ordre que celui des Africains $\mathrm{du}$ Copperbelt. Pour les Chagga, le tribalisme est une catégorie politique: leur chef représente le peuple face aux autorités extérieures. Au Copperbelt, le tribalisme est une catégorie des relations quotidiennes. Il fournit un mécanisme pour organiser les relations sociales avec des étrangers en réponse aux nécessités qui découlent d'une situation sociale fluide. Là où beaucoup d'hommes de différentes tribus sont concentrés sur un espace restreint, les syndicats ouvriers, le Congrès National Africain, et d'autres organisations similaires opèrent au même niveau de l'espace social que les Chagga et ils réunissent les Africains en opposition aux Européens sans se soucier de leur origine tribale. [...] 


\section{Le kalela en situation urbaine}

64

Nous sommes maintenant en mesure de retourner au paradoxe apparent qui à l'origine avait attiré mon attention sur la danse du kalela. Il convient de rappeler que l'un des traits marquants de la danse du kalela consistait dans le fait qu'elle était indubitablement une danse tribale, dans le sens où l'équipe était composée principalement de membres de la tribu Bisa et que leur but consistait en l'éloge des Bisa en général et de leur chef Matipa en particulier. Mais l'habillement qu'ils portaient et le langage qu'ils utilisaient dans leurs chants servaient à réduire le caractère tribal de leur identité de manière à permettre leur amalgame dans la population africaine du Copperbelt prise dans son ensemble.

65

J'ai essayé de montrer dans ce texte qu'une des caractéristiques de la structure sociale de la population africaine du Copperbelt réside dans le fait que, à l'exception de ces équipes de danse, le tribalisme ne constitue pas la base sur laquelle s'organisent les groupes structurés. Il reste essentiellement une catégorie d'interaction dans les relations sociales occasionnelles. De la même manière les hiérarchies de prestige n'organisent pas les Africains de manière structurée. De concert avec le tribalisme, elles opèrent comme une catégorie d'interaction qui sert à la médiation des relations sociales au sein d'une société en transition. Ces deux principes d'agrégation sociale déterminent le comportement entre gens qui ne se connaissent pas, essentiellement dans les relations quotidiennes. Il est impossible de faire des généralisations sur la manière dont opèrent ces principes sans se référer à la situation sociale dans laquelle l'interaction prend place ${ }^{67}$. McCall parle de « collectivités qui ont commencé à réunir en entités communes des éléments provenant de tribus disparates» et mentionne comme exemples de ces dernières «les écoles, les églises, les syndicats, les partis politiques, les mouvements nationalistes, et les lieux de loisirs publics tels que les bars à bières et les terrains de football ». «Plus les Africains s'identifient avec ces groupes, poursuit-il, moins l'affiliation tribale devient importante ${ }^{68}$ . " L'exemple de la Rhodésie du Nord suggère que, dans certaines situations, les Africains ignorent soit les différences de classe, soit les différences tribales (ou les deux) alors que, dans d'autres situations, ces différences deviennent significatives. J'ai présenté un exemple qui tendait à montrer que, dans leur opposition aux Européens, les Africains ignorent leurs différences à la fois de "classe » et de tribu. Au sein d'une association tribale telles que celles trouvées en Rhodésie du Sud, je pourrais m'attendre à ce que les oppositions soient énoncées en termes de différences de «classe ». Je pourrais également m'attendre à ce que les dissensions au sein d'une association d'enseignants ou d'employés de bureau soient formulées en terme de tribalisme. Les mêmes personnes qui sont solidaires dans une situation peuvent être farouchement opposées dans une autre. Le fait que le tribalisme n'émerge comme une catégorie significative de l'interaction que dans certaines situations, peut aider à expliquer quelques-unes des contradictions apparentes que des observateurs perspicaces ont notées de temps à autre. Hellman écrit par exemple que l'élargissement des perspectives et l'accroissement des connaissances que la vie urbaine a apportés aux Africains « ont créé un indigène aux fidélités divisées. Il ressent l'unité du peuple Bantou comme totalité ; mais il ne s'est pas libéré du sentiment de supériorité tribale qui pousse chaque tribu à s'appeler elle-même, chacune à son tour, "Le Peuple"69". Hellman cite le cas des luttes tribales dans les quartiers pauvres où elle travaille, ainsi que dans les quartiers de mineurs soumis à la ségrégation, comme exemple des situations typiques au cours desquelles le tribalisme sert à diviser la population en 
groupes opposés. Son exemple d'une situation où les distinctions tribales sont minimisées est également significatif. «Il y a à Johannesburg, écrit-elle, le Centre Social pour les Hommes Bantous où toute mention d'appartenances purement tribales est désapprouvée, et où l'usage de l'anglais comme langue véhiculaire est assidûment encouragé, ceci dans la croyance qu'une langue commune aidera à fondre les indigènes des différentes tribus, chacune avec sa propre langue, dans une nation bantoue ${ }^{70}$.» Plus haut dans le même paragraphe elle écrivait : "L'Afrique du Sud blanche est intimidée par la menace que cette "nation" en train d'émerger représente pour sa propre sécurité ».

L'équipe de kalela, en ce qu'elle est entièrement constituée de Bisa et a éliminé toute possibilité de différenciation en " classes " par l'adoption d'un habillement propre à ceux qui occupent les plus hautes positions dans l'échelle de prestige, est en mesure de présenter un front uni face aux étrangers. Pour les spectateurs il n'y a là aucun paradoxe. Je pense que le fait que cela représente un paradoxe pour nous résulte de l'ambiguïté du concept de tribalisme. Prenons en compte ces manifestations de tribalisme. Les Chewa utilisent leurs spectaculaires danses masquées de la cérémonie du nyau dans leurs danses au Copperbelt. En Rhodésie du Sud où les membres de tribus se constituent en groupes qui prennent la forme de sociétés de funérailles et d'amicales, un titre et une constitution remplissent la même fonction. Il arrive donc que les Bisa, de la même manière qu'un certain nombre d'autres tribus des régions du nord de la Rhodésie du Nord, ne portent pas de vêtements distincts et spécifiques, qui leur permettraient d'exprimer leur unité. De là le fait qu'ils aient recours aux couplets d'éloges dans leurs chants. Mais les associations de funérailles et les groupes de danses tribales ne sont pas dirigés par un chef et un groupe d'aînés de la tribu. À la place, ils ont un comité avec un président ou un « roi » accompagné de secrétaires, de trésoriers et d'autres officiels, et mènent leurs affaires de la même manière que le ferait toute association européenne. La structure tribale rurale n'a pas de rapport immédiat avec la composition de l'équipe de danse et le symbole particulier que cette dernière utilise pour exprimer son unité n'est pas fixe.

Je soutiens que l'ensemble des relations au sein d'un groupe de membres d'une tribu dans le cadre de leur région d'origine rurale est très différent de l'ensemble des relations de ce même groupe transposé dans un contexte urbain. En zone rurale, les relations forment une partie d'un système tribal complet. Les membres de la tribu établissent leurs relations entre eux en termes de liens de parenté, d'appartenance à un clan et à un village.

Dans les villes, le modèle du système social est largement déterminé par le système industriel qui forme la base de leur existence et par les lois que le gouvernement a édictées afin de réglementer la vie des citadins. Dans la mesure où les villes trouvent le fondement de leur développement dans la production industrielle, « le lien monétaire qu'implique l'acquisition des services et des biens a remplacé les relations personnalisées comme fondement des liens sociaux. L'individualité est, dans ces circonstances, remplacée par des catégories ${ }^{71}$ » La "tribu» est devenue au Copperbelt l'une de ces catégories et c'est en ce sens seulement que le kalela est une danse « tribale ». 


\section{NOTES}

1. Créé en 1937, le RLI a été marqué par la forte influence intellectuelle de Max Gluckman (1911-1975) qui en fut le directeur de 1941 à 1947. Il regroupa les recherches de terrain et les personnes qui formèrent le noyau de l'« école de Manchester ». En 1964, lors de l'Indépendance de la Zambie, le RLI est devenu l'Institute of African Studies de l'Université de Zambie.

2. J. C. Mitchell, The Yao village, Manchester, Manchester University Press, 1956.

3. «Foreword", in J. C. Mitchell, Cities, Society, and Social Perception. A Central African Perspective, Oxford, Clarendon Press, 1987, p. V.

4. Voir J. C. Mitchell, ed., Social Networks in Urban Situations, Manchester, Manchester University Press, 1969.

5. Evans-Pritchard publia également un paper du Rhodes-Livingstone Institute en 1945 sur les Nuer, et associa Max Gluckman à ses recherches sur les systèmes politiques africains.

6. J. C. Mitchell, «The Situational Perspective ", in Cities, Society, and Social Perception..., op. cit., p. 1-33.

7. C'est au cours de cette reprise que deux sections ont été ajoutées au texte initial, sur la distance tribale et sur les relations à plaisanterie inter-tribale en ville. Ces deux sections ne figurent pas dans la présente traduction. En effet, faute de place suffisante pour publier la traduction de l'ensemble du texte, nous avons choisi d'écarter ces deux parties-ci : il s'agit de digressions dont l'absence n'empêche pas la compréhension du raisonnement de Mitchell et de la méthode suivie. N'ont pas été repris non plus, faute de place, dans la présente traduction, la section sur le tribalisme et l'administration urbaine, les annexes de tableau et les textes des chants. Nous remercions Manchester University Press de nous avoir autorisé à publier la présente traduction.

8. F. Barth, "Introduction», Ethnic Groups and Boundaries. The Social Organization of Culture Difference, Boston, Little Brown, 1969, p. 35.

9. U. Hannerz, Explorer la ville. Éléments d'anthropologie urbaine [1980], Paris, Minuit, 1983, p. $172-176$.

10. À l'occasion du Memorial Meeting en hommage à Clyde Mitchell, tenu le 24 février 1996 au Nuffield Collège, Oxford. Merci à Peter Wade, professeur au département d'anthropologie sociale de l'Université de Manchester, pour ses informations biographiques sur Clyde Mitchell et pour le compte rendu du Memorial Meeting.

11. M. Gluckman, "Analysis of a Social Situation in Modern Zululand», Bantu Studies, XIV, mars 1940, p. 1-30, et juin 1940, p. 147-174.

12. E. Jokl, "Physical Education, Sport and Recreation ", in E. Hellman et L. Abrahams, eds, Handbook of Race Relations in South Africa, Cape Town, Oxford University Press, 1949, p. 412 sq. Un prix est décerné chaque année à Salisbury à l'équipe qui gagne la compétition de danse tribale. En 1954 il a été gagné par une équipe Shangaan. Report of the Director of Native Administration of Salisbury for the Year ending June 1955, para. 273, p. 54.

13. II existe beaucoup de danses très similaires au kalela, mais qui portent des noms différents. La danse mbeni, qui s'était répandue en Afrique centrale avant la guerre de 1939-1945, et dont mes informateurs m'ont dit qu'en était dérivé le kalela, était à peu près identique. Une danse connue sous le nom de mganda dans la Province de l'Est de la Rhodésie du Nord lui ressemble également. Une danse appelée malipenga des Tonga de Lakeside possède beaucoup de caractéristiques semblables. M.C.M.N. White me dit qu'une danse similaire est connue chez les Luvale, les Luchazi et les Chokwe sous le nom de nyakasanga, tout en faisant remarquer que leurs voisins, les Lunda 
de l'Ouest, appellent leur danse kalela. Au cours d'une compétition récente de danses tribales qui s'est tenue à Mufulira et dont rend compte le Mufulira Star, vol. 4 (6) (juin 1956), les Kalela Smart et les Karonga Bonna, qui se sont classés respectivement deuxième et troisième, sont des équipes de danseurs de type kalela. W. V. Brelsford (African Dances of Northern Rhodesia, Occasional papers of the Rhodes-Livingstone Museum, New Series, 2, Livingstone, Rhodes/Livingstone Museum, 1948) ne mentionne pas le kalela.

14. Je suis redevable à $M$. Sykes Ndilila, à cette époque assistant de recherche au RhodesLivingstone Institute, pour ce qui concerne la collecte des informations personnelles sur les danseurs ainsi que pour l'enregistrement des paroles des chants.

15. On retrouve l'usage de ces titres officiels « européens » dans des groupes de danse urbains en Afrique de l'Ouest. Cf. M. Banton, « Ambas Geda », West Africa, 24 octobre 1953.

16. II y a aussi un homme qui dans l'équipe se dit « Ngoni ». Il est le fils d'un membre de la tribu Yao né à Fort Jameson et est le « meilleur ami » de Luke. Apparemment il doit son appartenance à l'équipe à une faveur spéciale.

17. Dans les chants où ils évoquent l'unité bisa, ils nomment leur chef suprême « le commissaire de Province Kopa » [N.d.T.].

18. II n'y avait qu'un danseur parmi les dix-neuf qui était âgé de trente ans, alors que dans la population globale de Luanshya, 47,5\% des hommes adultes étaient âgés de trente ans et plus. Voir J.C. Mitchell, African Urbanization in Ndola and Luanshya, Lusaka, Rhodes-Livingstone Communication, 6, 1954, tableau I, p. 4.

19. Version africaine des Témoins de Jehova implantée en Rhodésie depuis la fin du XIX siècle [N.d.T.].

20. Voir par exemple le compte rendu d'une compétition de danse kalela dans African Roan Antelope, II, décembre 1953, p. 6, où l'on trouve des observations particulières sur l'élégance des habits des danseurs.

21. Vraisemblablement du verbe bemba ukuwela: huer ou se moquer (Bemba-English Dictionary, Chilubula, White Fathers, 1947).

22. Nous savons que le mbeni existait dans le Copperbelt en 1935 mais nous ne savons pas s'il disparut à cette époque ou s'il persista et fut absorbé ultérieurement par le kalela.

23. Parmi les Yao dans les districts de Liwonde et de Fort Johnston, en 1946-1949, en tant qu'assistant anthropologue au Rhodes-Livingstone Institute.

24. Mon informateur attira mon attention sur le fait que les tambours utilisés étaient des "tambours européens », c'est-à-dire qu'ils étaient à double face et contrefaisaient de cette manière la grosse caisse militaire. Ce qui contraste naturellement avec le tambour traditionnel fait à partir d'un tronc d'arbre creux recouvert d'une peau à l'une de ses extrémités. Cette explication concernant l'origine du beni, ainsi que beaucoup des détails décrits par mon informateur sont confirmés par une note concernant la danse rédigée par le commissaire en chef de la police de Zomba, Nyasaland, en 1921. Voir le dossier N3/23/2 aux Central African Archives, Salisbury, Rhodésie du Sud.

25. C'est ainsi que mon informateur me l'a énoncé. Il ne semblait pas conscient de l'inversion de rang.

26. Mon informateur commenta avec un sourire forcé que quelques-uns avaient été poursuivis en justice pour le vol de ceinturons Sam Browne.

27. Evidence taken by the Commission appointed to Enquire into Disturbances in the Copperbelt, 1935 (Russell Commission), Imprimerie du Gouvernement de Rhodésie du Nord, Lusaka, 1935, p. 77. Dans sa note datée du 27 juillet 1921, le commissaire en chef de la police du Nyasaland rapportait que ces danses avaient fleuri dans les colonies allemandes de l'Afrique de l'Est avant la guerre de 1914-1918 et que les titulaires de la fonction principale au sein de celles-ci portaient des titres allemands tels que "Kaiser, Kaiserin, Hauptmann », etc. (dossier N3/23/2 aux Central African Archives). La description que Jones fait de la danse Mganda correspond exactement à celle de la 
danse mbeni; il dit que c'était franchement une parodie de parade militaire qui était parvenue d'Afrique de l'Est pendant la guerre de 1914-1918 et avait été introduite en Rhodésie du Nord par des Tonga de Lakeside :

A. M. Jones, «African Music : the Mganda Dance », African Studies, IV (4), 1945, p. 180-188.

28. La commission Russell découvrit que quelques leaders de la Société Mbeni se trouvaient impliqués dans les troubles mais que, en tant que telle, la Société n'était pas subversive. Rapport de la Commission Russell, p. 49.

29. W. V. Brelsford, African Dances of Northern Rhodesia, op. cit., p. 19.

30. Jones, par exemple, mentionne l'exécution quelque peu terne d'une danse similaire dans la région rurale de Fort Jameson. A. M. Jones, « African Music : the Mganda Dance », op. cit.

31. Les Africains étaient admis au sein de l'armée en tant que soldats et sous-officiers et, naturellement, comprenaient le système des grades militaires.

32. Une amusante variante de ce constat, qui m'a été rapportée par M.E. Tikili, collaborateur scientifique du Rhodes-Livingstone Institute, est que les Tonga de Lakeside, qui ont leur propre version du mbeni qu'ils appellent malipenga, portent des kilts lorsqu'ils dansent à Bulawayo. Les premiers Européens à avoir vécu dans le pays des Tonga de Lakeside, à Livingstonia, étaient des Écossais. J. van Velsen, responsable de recherche au Rhodes-Livingstone Institute, actuellement en train d'effectuer un travail de terrain parmi les Tonga de Lakeside, a décrit une danse malipenga ayant eu lieu à Chinteche. Là il n'y avait pas de kilts, mais J. van Velsen décrit la danse comme ressemblant à un "gymkhana » dans lequel la caractéristique principale est la mise élégante des participants.

33. Témoignage devant la commission Russell, p. 77.

34. Cf. B. Barber, "Acculturation and Messianic Movements", American Sociological Review, XI, 1946, p. 663-669.

35. II convient de noter que dans le Règlement des Townships (Contrôle des Indigènes) (chap. 120 des Lois de la Rhodésie du Nord), section 7, personne n'est autorisé à organiser ou à participer à une quelconque danse dont les intentions délibérées sont de ridiculiser ou de mépriser toute personne, religion ou autorité dûment constituée. Je ne pense pas que les danseurs de kalela soient au courant d'un tel règlement.

36. A. M. Jones, « African Music : the Mganda Dance », op. cit.

37. Voir la note 20, p. 223.

38. Un journal publié pour le personnel africain de la compagnie Nkana rapporte le déroulement d'un concours vestimentaire gagné par un vendeur de magasin. Le second était un aideinfirmier : Luntandanya, II, 1, mai 1954, 3. De telles compétitions avaient également lieu dans les villes d'Afrique du Sud. Le professeur Gluckman m'a dit qu'il avait officié comme membre du jury dans une de ces compétitions, qui s'était déroulée dans le cadre d'une soirée à Pretoria en 1937, soirée durant laquelle avait lieu un concours de danses européennes. La plupart des personnes présentes étaient des domestiques. Lorsqu'il sélectionna l'homme le mieux habillé, un autre compétiteur protesta qu'il n'avait pas examiné les sous-vêtements, et il lui fut demandé de le faire.

39. G. Wilson, An Essay on the Economics of Detribalization in Northern Rhodesia, t. 2, Livingstone, Rhodes-Livingstone Paper, 6,1942, p. 18. Il a découvert que 67,4\% des dépenses en argent des Africains en dehors de la nourriture étaient consacrées, dans le complexe des mines de Broken Hill en 1939-1940, à l'habillement. Dans un échantillon de Mufulira et de Chingola en 1953, le pourcentage était de 40,6. Cf. A. A. Nyirenda, African Market Vendors in Lusaka, 1956.

40. G. Wilson, An Essay on the Economics of Detribalization in Northern Rhodesia, op. cit., p. 15.

41. B. Gussman (African Life in an Urban Area, Bulawayo, Federation of African Welfare Societies, 1952, p. 57) fait un constat similaire en décrivant Bulawayo en 1950. Il fait remarquer qu'il n'y a que peu d'autres possibilités accessibles aux Africains d'investir l'argent qui leur reste. 
42. G. Wilson, An Essay on the Economics of Detribalization in Northern Rhodesia, op. cit., p. 15. Plusieurs années avant, Hunter avait fait la même remarque au sujet des Africains d'East London en Afrique du Sud. Elle écrivait ceci : «Dans les villes il est chic d'être aussi européanisé que possible. Le statut dépend largement de la richesse et de l'éducation et celles-ci impliquent l'européanisation ", M. Hunter, Reaction to Conquest. Effects of Contact with Europeans on the Pondo of South Africa, Londres, Oxford University Press for the International African Institute, 1936, p. 437. 43. Les Africains expriment leurs aspirations précisément en ces termes. Une des raisons principales de l'attraction des villes est qu'elles représentent une occasion pour les Africains d'« acquérir de la civilisation » (ukukwata shifilaiseshoni). Little fait la même observation en ce qui concerne les Mende de Sierra Leone. Cf. K. Little, «Social Change and Social Class in the Sierra Leone Protectorate ", American Journal of Sociology, LIV (1), 1948, p. 15-16 et « Structural Change in the Sierra Leone Protectorate ", Africa, XXV (3), 1955, p. 217-233.

44. Conduite auprès de 653 écoliers, étudiants et enseignants-stagiaires à Lusaka par A. I. Epstein et moi-même. Elle sera publiée intégralement en temps utile.

45. R. Merton et P. F. Lazarsfeld, Continuities in Social Research, Glencoe, The Free Press, 1950 ; également

J. C. Mitchell, "The African Middle Classes in British Central Africa », papier soumis au INCIDI, Conference on the Development of the Middle Classes in Tropical Countries, Londres, 1955.

46. En français dans le texte [N.d.T.].

47. En français dans le texte [N.d.T.].

48. G. C. Coulter, "Sociological Aspects", in D. Merle, ed., Modern Industry and the African, Londres, Macmillan, 1933, p. 86.

49. Ce point a été aussi soulevé par Hunter à propos de la population africaine dans une ville d'Afrique du Sud. Elle écrivait: «Le clivage entre Bantous et Européens augmentait la solidarité entre Bantous (et entre Européens) et éclipsait les différences économiques au sein de la communauté Bantoue elle-même ", M. Hunter, Reaction to Conquest..., op. cit., p. 465.

50. M. McCulloch, A Social Survey of the African Population of Livingstone, Rhodes-Livingstone Paper, 26, Manchester, Manchester University Press, 1956, p. 50.

51. Principalement originaires des provinces du Nord et de l'Est de la Rhodésie du Nord.

52. M. McCulloch, A Social Survey..., op. cit., table 23.

53. F. Spearpoint, "The African Natives and the Rhodesian Copper Mines ", Supplement to the Journal of the Royal African Society, XXVI, 1937, p.16-18. Voir également le rapport de la commission Russell.

54. Le banc des magistrats est composé de quatre ou cinq assesseurs originaires des chefferies rurales dont les tribus sont numériquement prépondérantes en ville. Ils fonctionnent comme une cour mineure et auditionnent la plupart des cas concernant « les lois coutumières indigènes ». Cf. A. L. Epstein, The Administration of Justice and the Urban African, Londres, Colonial Research Series, 1953.

55. G. Wilson, An Essay on the Economics of Detribalization in Northern Rhodesia, op. cit., p. 75.

56. Le parallèle avec les amicales qui se sont développées au sein des classes ouvrières urbaines en Angleterre pendant la révolution industrielle est frappant. Cf. J.-L. et B. Hammond, The Bleak Age, Londres, Pelikan Books, p. 227-228.

57. Voir p. 45, 195.

58. M. McCulloch rapporte qu'à Livingstone en 1953, il y avait environ 27 associations tribales.

M. McCulloch, A Social Survey..., op. cit., p. 8.

59. Luntandanya, II, 8, novembre 1954.

60. J. C. Mitchell, «The Distribution of African Labour by Area of Origin on the Coppermines of Northern Rhodesia ", in Human Problems in British Central Africa, XIV, 1954, p. 30-36.

61. Les chambres des logements de célibataires ont tendance à être occupées par quatre à six hommes du même groupe ethnique. G. Wilson, An Essay on the Economics of Detribalization in 
Northern Rhodesia, op. cit., p. 75, rapportait en 1940 qu'à Broken Hill « il existe une tendance pour les membres de même tribu à être regroupés dans des cases adjacentes ». Je ne sais pas si c'est toujours vrai.

62. Je remercie le Dr W. Watson et M. J. van Velsen qui m'ont rapporté cet incident.

63. N. S. Shaler, The Neighbour, New York, Houghton Mifflin and Co., 1904. Repris dans R. E. Park et

E. W. Burgess, Introduction to the Science of Sociology, Illinois, University of Chicago Press, 1921, p. 295.

64. E. T. Hiller, Social Relations and Structures, New York, Harper Bros, 1947, p. 643.

65. V. Harlow, « Tribalism in Africa », Journal of African Administration, VII, 1955, p. 17-20.

66. Ibid., p. 19. C'est moi qui souligne.

67. Cf. M. Gluckman, Custom and Conflict in Africa, Oxford, Basil Blackwell, 1955, p. 151-163.

68. D. F. McCall, «Dynamics of Urbanization in Africa », p. 158, in Annals of the American Academy of Political and Social Science, 298, 1955, p. 151-160.

69. E. Hellman, Rooiyard. A Sociological Survey of an Urban Native Slum Yard, Rhodes-Livingstone Paper, 13, Cape Town, Oxford University Press, 1948, p. 114.

70. Ibid.

71. L. Wirth, "Urbanism as a Way of Life », American Journal of Sociology, XLIV, 1938, réédité in P. K. Hatt et A. J. Reiss, Reader in Urban Sociology, Glencoe, The Free Press, 1951, p. 44.

\section{RÉSUMÉS}

La danse du kalela est exécutée, aux heures de loisir, par des citadins africains d'une ville minière d'Afrique australe, dans les années cinquante. Elle se réfère au monde supérieur des Blancs (par la tenue des danseurs) et, en même temps, aux relations inter-ethniques africaines (par les thèmes des chants et par le recrutement ethnique des groupes de danse). Cette double référence sert de fil conducteur à l'analyse des relations sociales (parmi les Africains et entre Blancs et Noirs) dans le Copperbelt. Le tribalisme s'avère ainsi être une option urbaine de classement social dans certaines situations.

The kalela dance was performed during leisure time by African town dwellers of a mining city of austral Africa in the 1950's. It refers to the superior world of the Whites (through the dancers costumes) and to inter ethnic African relationships at the same time (through the themes of songs and the ethnic recrutement of the groups of dance). This double reference serves as a clue for the analysis of social relationships (between Africans and between Whites and Blacks) in the Copperbelt. Tribalism thus proves to be an urban option of social classification in certain situations. 